\title{
OTIMIZAÇÃO DO SALDO DE CAIXA COM ALGORITMOS GENÉTICOS: UM ESTUDO RELACIONANDO CRUZAMENTO E MUTAÇÃO NO MODELO DE MILLER E ORR
}

\section{CASH BALANCE OPTIMIZATION WITH GENETIC ALGORITHMS: A STUDY OF CROSS AND MUTATION RELATIONSHIP IN MILLER-ORR MODEL}

\author{
Marcelo Botelho da Costa Moraes* E-mail: mbotelho@usp.br \\ Marcelo Seido Nagano* E-mail: drnagano@usp.br \\ *Universidade de São Paulo, São Paulo, SP
}

\begin{abstract}
Resumo: O presente trabalho tem por objetivo a aplicação de algoritmos genéticos na definição de políticas de administração do saldo de caixa. Este problema de finanças abordado inicialmente por Baumol (1952) e Tobin (1956) teve sua origem na aplicação de modelos determinísticos de controle de inventário ao caixa existente nas empresas. Posteriormente Miller e Orr (1966) aperfeiçoam a abordagem ao introduzirem um modelo estocástico que não mais definia o ponto ideal do saldo de caixa, mas uma faixa de oscilação. Este trabalho propõe uma metodologia baseada em modelos evolutivos, com algoritmos genéticos, em diferentes abordagens, para otimizar o saldo de caixa, utilizando para isso premissas apresentadas na literatura. Para tal são utilizadas simulações no apoio e validação do modelo. Os resultados indicam que os algoritmos genéticos podem ser muito úteis na parametrização do modelo de Miller e Orr, apresentando resultados promissores neste tipo de problema, sendo a solução ótima fortemente associada ao processo evolutivo de mutação. Ficam perspectivas futuras para uma melhor aplicação dos algoritmos genéticos em problemas de otimização do saldo de caixa.
\end{abstract}

Palavras-chave: Otimização. Saldo de Caixa. Modelos Computacionais. Gestão de Tesouraria. Miller-Orr.

\begin{abstract}
This work has the objective to apply genetic algorithms in cash balance policy definition. This financial problem was initially treated by Baumol (1952) and Tobin (1956) which work applies deterministic models to inventory control in enterprises cash balance. Further, Miller and Orr (1966) enhanced the problem approach introducing a stochastic model which no longer defines the optimal cash balance, but bands of oscillation. This work proposes an evolutionary model methodology with genetic algorithms, in different approaches to cash balance optimization, using the premises showed in literature. For this, simulations are used in model support and validation. The results shows that genetic algorithms can be very useful in Miller-Orr model parameterization, with good results in this field of problem, and the optimal solution has a strong association with mutation evolutionary process. This paper let future perspectives of better application of genetic algorithms in cash balance optimization problem.
\end{abstract}

Keywords: Optimization. Cash Balance. Computational Models. Treasury Management. Miller-Orr. 


\section{INTRODUÇÃO}

Gerenciar o saldo disponível de caixa é um problema constante em todo o tipo de organização. Isto ocorre em função das entradas e saídas diárias de dinheiro, sejam elas pela atividade operacional da empresa ou por operações financeiras que esta tenha negociado. Assim, existe a necessidade de controlar os recursos financeiros de maneira a obter o melhor resultado para a organização.

Nesse sentido, a função da administração de caixa tem como responsabilidades mobilizar, controlar e planejar os recursos financeiros das empresas (SRINIVASAN; KIM, 1985). Com isso, a utilização de modelos de apoio a tomada de decisão se torna pertinente, uma vez que podem proporcionar uma visão abrangente e de otimização algo que dificilmente pode ser obtido sem a utilização de metodologias para tal.

O saldo de caixa consiste no dinheiro disponível, em determinado momento no tempo, para a organização. Ele é afetado constantemente pelas entradas e saídas de dinheiro, provenientes dos recebimentos e resgates de aplicações financeiras como entradas e pagamentos e investimentos financeiros como forma de saídas de recursos, ambos realizados pela organização. Assim, o saldo de caixa é o resultado de um saldo de caixa em uma data anterior, modificado pelo fluxo líquido de caixa ocorrido.

Dessa forma, a utilização de modelos no problema de definição do nível ideal de recursos disponíveis em caixa teve sua origem nos trabalhos de Baumol (1952) e Tobin (1956), onde os autores partem do pressuposto de que saldo disponível em caixa pode ser definido como uma commodity em estoque. Ou seja, o caixa seria um bem padronizado com controle diário, semanal, mensal, etc., dependendo do nível de detalhamento temporal exigido pela empresa.

Para estes autores, a definição do saldo de caixa ótimo segue o padrão dos modelos de dimensionamento de estoque, onde se considera o recurso financeiro disponível como um estoque, que possui certos custos associados a sua origem e manutenção, mas que também gera benefícios indispensáveis para a organização.

Dessa forma, a definição do saldo de caixa passou a ter uma abordagem quantitativa no intuito de promover a otimização deste estoque financeiro, de modo a minimizar os custos associados à manutenção ou falta de dinheiro em caixa. 
Posteriormente, Miller e Orr (1966, p. 415) definem o saldo de caixa como tendo uma flutuação irregular, se caracterizando como uma variável aleatória e propõem um modelo estocástico para o gerenciamento do saldo de caixa.

Segundo Sá (2008), em um mercado perfeito, com ausência de impostos, custos de transação e limitações ao crédito, além da simetria de informação (pressuposto de mesma informação disponível a todos), a rentabilidade de uma empresa não seria afetada pela forma como seus recursos são alocados, ficando o saldo de caixa irrelevante, desde que suficiente para liquidar os compromissos no seu vencimento.

Já no mundo real, os mercados não são perfeitos, e a sobra de recursos financeiros, também conhecida como liquidez, possui um custo associado. Nesses casos, a estratégia ótima para uma empresa é reter dinheiro, prevendo sazonalidades na demanda por seus produtos e choques aleatórios que possam afetar seus negócios (BERK; DEMARZO, 2008).

Assim, entender os motivos que levam as organizações a possuir a necessidade de manter recursos em caixa é fundamental para uma melhor gestão financeira. Nesse sentido, Braley e Myers (apud SÁ, 2008) apontam quatro motivos para a manutenção do saldo de caixa:

1. Transações - recursos mantidos em caixa para honrar compromissos em vista do descompasso temporal entre as saídas (pagamentos) e as entradas (recebimentos) de dinheiro;

2. Precaução - recursos mantidos líquidos em caixa como manutenção de uma reserva de segurança para contingências;

3. Especulação - recursos mantidos em caixa para aproveitar oportunidades de obtenção de descontos ou aplicações favoráveis; e

4. Reciprocidade bancária - recursos mantidos em contas correntes para atender a exigências de alguns bancos como contraprestação.

Ainda assim, a definição da quantidade de dinheiro a ser mantida em caixa não é algo tão facilmente compreendida ou realizada. Outro fator de relevância na definição de políticas de gerenciamento do saldo de caixa depende de fatores restritivos. 
No caso brasileiro, dados da Economática referentes ao período de 2004 a 2008 indicam que as empresas brasileiras (de atividade não financeira) com ações negociadas em bolsa, obtiveram um saldo médio ponderado de disponibilidades de $8,85 \%$ no período (Tabela 1 ).

Tabela 1 - Participação das disponibilidades no total dos ativos - empresas brasileiras

\begin{tabular}{lccccc}
\hline Brasil & $\mathbf{2 0 0 8}$ & $\mathbf{2 0 0 7}$ & $\mathbf{2 0 0 6}$ & $\mathbf{2 0 0 5}$ & $\mathbf{2 0 0 4}$ \\
\hline \% Disponível (Médio) & $9,10 \%$ & $11,39 \%$ & $9,22 \%$ & $7,49 \%$ & $6,75 \%$ \\
Desvio-padrão & $15,81 \%$ & $17,35 \%$ & $16,27 \%$ & $14,72 \%$ & $13,87 \%$ \\
Quantidade Empresas & 567 & 369 & 366 & 350 & 353 \\
\hline
\end{tabular}

Fonte: Economática (2009)

Dessa forma, o trabalho busca atender a metodologia quantitativa de determinação do nível de caixa ótimo, utilizando o modelo proposto por Miller e Orr sem a necessidade de pressupor qual o valor mínimo (limite inferior) que o saldo de caixa pode chegar.

Com o intuito de atender ao problema proposto, tem-se como objetivo geral da pesquisa desenvolver uma metodologia de otimização do saldo disponível de caixa, com base nas premissas de minimização do custo.

Para alcançar o objetivo proposto a seguinte metodologia quantitativa é utilizada:

- Simular séries históricas de fluxos de caixa, com base em premissas observadas na literatura sobre o tema;

- Desenvolver o modelo de algoritmos genéticos que utilizem o modelo de Miller e Orr e tenham como função objetivo a otimização do saldo de caixa em relação ao limite inferior;

- Aplicar o modelo desenvolvido nas simulações de fluxos de caixa e analisar seus resultados.

\section{REFERENCIAL TEÓRICO}

\subsection{Modelos de Administração de Caixa}


Os modelos de administração de caixa tiveram sua origem no trabalho de Baumol (1952), nele o autor faz um paralelo entre o caixa com os demais estoques das empresas. Observando o modelo geral de estoque, tem-se um sistema onde o nível de um item (Figura 1) é afetado por um processo de entrada e por um processo de saída. Onde $\mathrm{P}(\mathrm{t})$ é a taxa em que o ativo é adicionado ao estoque no tempo $\mathrm{t} e$ $W(t)$ é a taxa em que o ativo é reduzido do estoque (BEDWORTH e BAILEY, 1987). Normalmente se assume que a saída ocorre em função de uma demanda $D(t)$, consistindo em uma força externa.

Figura 1 - Um sistema de estoque

Demanda, $D(t)$

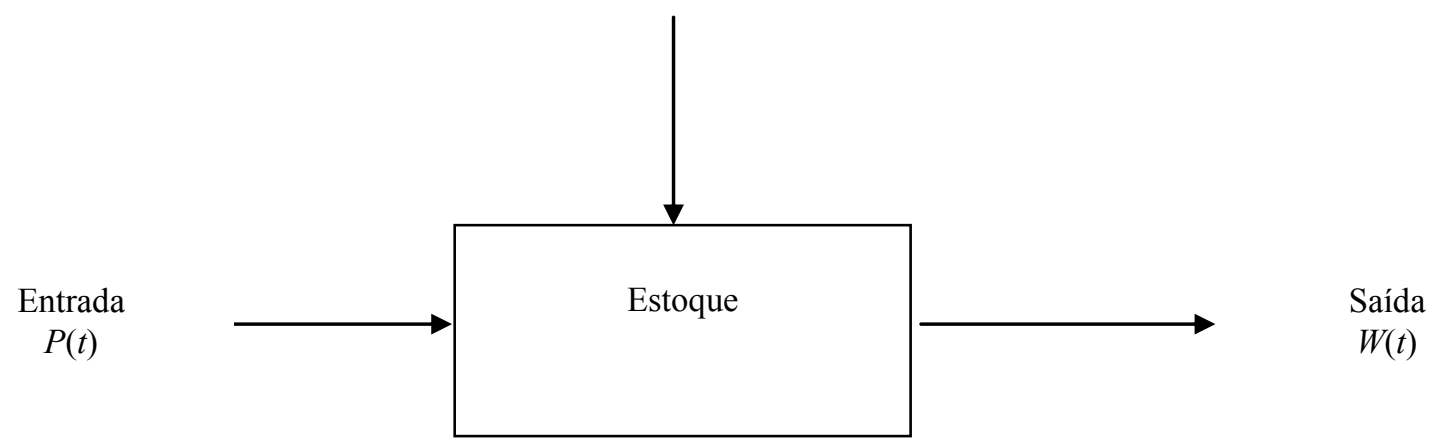

Fonte: Bedworth; Bailey (1987)

No caso dos estoques em geral a abordagem mais comum quando se necessita definir o reabastecimento do estoque é o lote econômico de compra (LEC), que vise encontrar o melhor posicionamento entre as vantagens e desvantagens de possuir estoque.

Apesar disso, o LEC (Figura 2) possui restrições ao utilizar os pressupostos de demanda fixa e previsível, bem como entregas instantâneas quando da solicitação da reposição do estoque (SLACK et al, 1997, p. 388). 
Figura 2 - Perfis de estoque que ilustram a variação do nível de estoques.

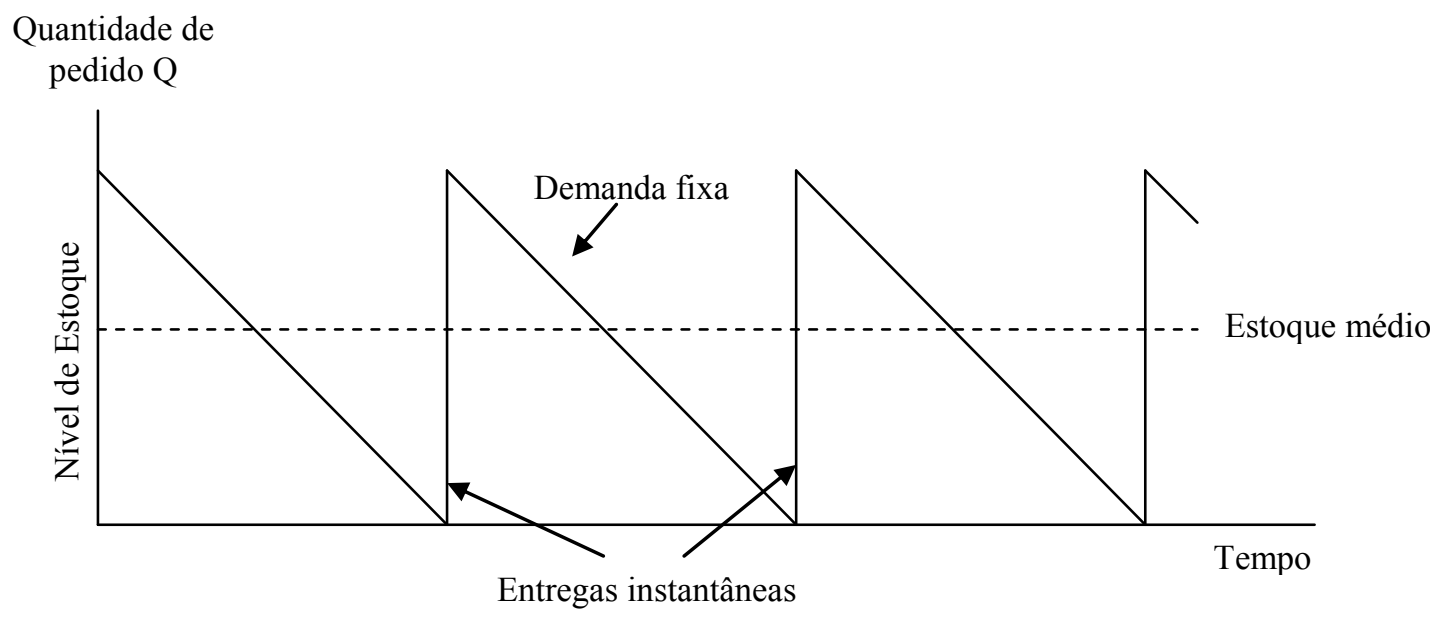

Fonte: Adaptado de Slack et al. (1997)

Segundo Baumol (1952) o estoque de caixa pode ser observado como um inventário de um meio de troca. Nesse modelo adaptado do LEC para otimização de caixa a configuração ótima é obtida em função da relação entre o custo de oportunidade e o custo de transação. No modelo o custo de transação aumentam quando a empresa precisa vender títulos para acumular caixa, já os custos de oportunidade crescem com a existência do saldo de caixa, pois é uma aplicação que não possui rendimento (ROSS; WESTERFIELD; JAFFE, 2002).

O modelo efetua a análise do custo associado à manutenção de dinheiro em caixa, ou seja, o custo de oportunidade determinado pelos juros que a empresa deixa de receber ao não aplicar os recursos, e do custo de obtenção do dinheiro pela conversão dos investimentos em caixa (ASSAF NETO, 2005), já o custo de transação representa os dispêndios incorridos na aplicação/resgata dos recursos financeiros, como taxas e impostos.

Segundo Assaf Neto (2005) o modelo de Baumol pode ser apresentado isoladamente da seguinte forma:

$$
\text { Custo de obtenção }=b \times \frac{T}{C}
$$

Onde, $b=$ custo fixo identificado nas transações (investimento ou resgate) em percentual;

$\mathrm{T}$ = valor total de caixa (em \$) que se prevê utilizar em determinado período em seu valor líquido, ou seja, a sobra do fluxo de caixa; 
$\mathrm{C}=$ saldo monetário em caixa (em \$).

$$
\text { Custo de manutenção }=i \times \frac{C}{2}
$$

Sendo, i = taxa de juros definida pela aplicação financeira;

C / 2 = saldo médio de caixa, admitindo-se que seu volume se reduza a uma taxa constante (demanda fixa) em unidades monetárias.

Com o cruzamento das duas retas, igualando-se as fórmulas, obtém-se o valor ótimo de caixa " $\mathrm{C}$ ", sendo ele:

$$
C^{*}=\sqrt{\frac{2 \times b \times T}{i}}
$$

Posteriormente, Miller e Orr (1966) apresentam a um modelo que atende a aleatoriedade dos fluxos de caixa, apesar de ainda considerar a existência de apenas dois ativos, caixa e investimento, sendo este último representa uma opção de baixo risco e alta liquidez (Figura 3).

Figura 3 - Variação dos fluxos de caixa Caixa (\$)

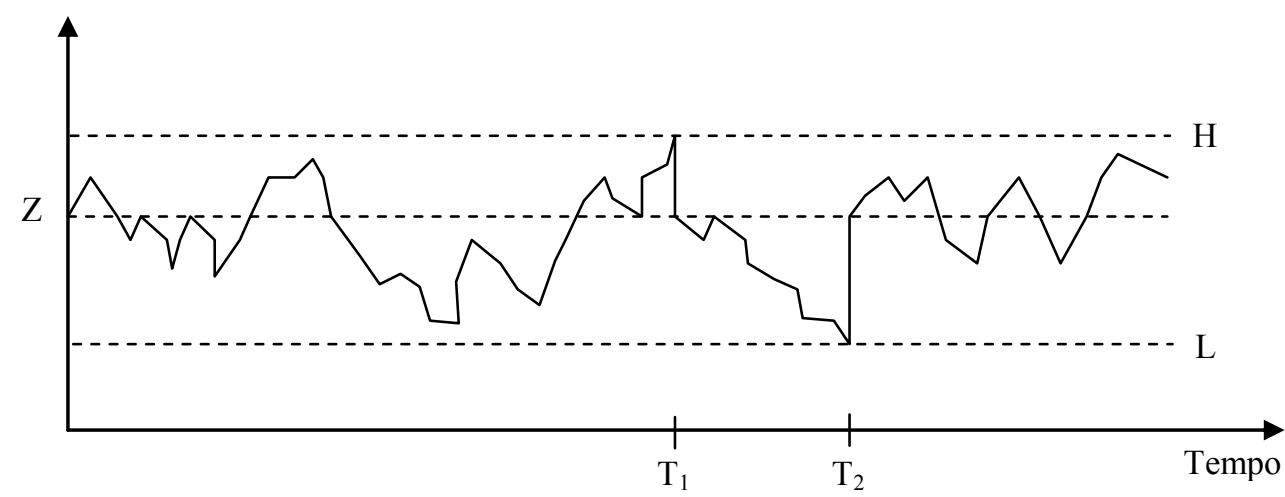

Fonte: Adaptado de Miller; Orr (1966)

Neste modelo procura-se definir dois limites para o nível de recursos em caixa: o mínimo e o máximo, de tal forma que, ao se atingir o nível máximo (T1), representado pelo limite superior $(\mathrm{H})$, efetua-se aplicação de recursos, em valor que leve o saldo de volta ao nível ideal de caixa ( $Z$ ), e ao se atingir o nível mínimo (T2) no limite mínimo (LM), procede-se no resgate, para obter o nível ótimo de caixa novamente (MATIAS, 2007). 
Assim, ao trabalhar os fluxos líquidos de caixa (entradas menos saídas) o modelo de Miller e Orr possibilita a otimização do caixa, com base nos custos de transação (representados por F) e oportunidade (representados por K), obtendo-se a seguinte formulação (ROSS; WESTERFIELD; JAFFE, 2002):

$$
Z^{*}=\sqrt[3]{3 F \sigma^{2} / 4 K+L}
$$

Sendo que o "*" denota valores ótimos e $\sigma^{2}$ é a variância dos fluxos líquidos de caixa. Mesmo com o ganho em relação ao modelo de Baumol, por considerar a aleatoriedade dos fluxos de caixa, o modelo de Miller e Orr pressupõe a definição do limite inferior (LM), ou seja, o risco de falta de caixa, associado a uma margem mínima de segurança, depende de uma escolha da administração e não é tratado no modelo.

Neste ponto recai o problema abordado neste trabalho, uma vez que o modelo de Miller e Orr não define por si só o limite inferior, cabe a utilização de algoritmos genéticos neste problema de definição do limite inferior ótimo, que seja capaz de minimizar o custo.

Posteriormente, a maioria dos trabalhos desenvolvidos utilizam os mesmos pressupostos dos modelos originais, principalmente de Miller e Orr, se diferenciando por uma modelagem estocástica do problema, como nos trabalhos desenvolvidos por Tapiero e Zuckerman (1980), Milbourne (1983), Hinderer e Waldman (2001), Baccarin (2002), Premachandra (2004), Volosov et al (2005), Liu e Xin (2008) e Baccarin (2009).

Poucos trabalhos utilizam uma metodologia computacional para resolução do problema, como a proposta de Yao, Chen e Lu (2006) que aborda sistemas fuzzy, bem como Gormley e Meade (2007) na utilização de algoritmos genéticos.

\subsection{Algoritmos Genéticos}

Os modelos genéticos fazem parte da família dos modelos evolutivos que possuem sua origem na inteligência artificial. A inteligência artificial é um campo que busca o desenvolvimento de sistemas que sejam capazes de tomar decisões com base no raciocínio. Assim, esses sistemas computadorizados devem aprender de acordo com uma base de informação e serem capazes de extrapolar esta informação, generalizando a aplicação desse conhecimento a novas possibilidades. 
Existem duas formas de aprendizado, 1) a dedutiva na qual o sistema é desenvolvido baseado em regras que tornam possível o raciocínio por meio da generalização do conhecimento, normalmente o processo de formalização do conhecimento é realizado por especialistas; e 2) a indutiva na qual são aplicadas técnicas de extração do conhecimento (conhecidos por indutores) sobre uma base de dados passados, sendo obtidos modelos sob a forma de regras, algoritmos ou funções matemáticas capazes de gerar o conhecimento.

Os modelos indutivos podem ter seu aprendizado supervisionado, no qual o erro do modelo é controlado, pois são conhecidas todas as características dos dados históricos utilizados em seu aprendizado, ou não supervisionado, onde apenas se tem a informação histórica, sem sua classificação. Diferentes modelos indutivos apresentam diferentes vieses (bias) em seu resultado (ZHANG; TSAI, 2003).

Normalmente as metodologias de modelos computacionais evolutivos buscam a otimização de um problema definido por meio de uma formulação matemática de seu objetivo, denominada função objetivo. Os modelos evolutivos são um caso especial em termos de aprendizado, pois ao mesmo tempo em que dependem do aprendizado dedutivo na formulação de sua função objetivo também se utilizam de algoritmos indutivos para obtenção da otimização esperada.

A computação evolutiva tem sua origem no estudo da teoria de evolução natural, sendo modelos de algoritmos que buscam atingir funções objetivos definidas, para isso, partem de possibilidades de resolução aleatórias e de acordo com seu algoritmo de desenvolvimento evoluem no sentido de obter melhores resultados na busca do objetivo estabelecido (REZENDE, 2005).

Para se tornarem úteis, os algoritmos tradicionais de descoberta das solucionais mais apropriadas, ou algoritmos de otimização, utilizam uma séries de suposições ou hipóteses sobre como avaliar a aptidão de uma solução. Outra forma tradicional de otimização, baseada em gradientes-descendentes depende da ocorrência de baixas oscilações no problema sob pena de obter uma otimização local e não global.

Mas os algoritmos evolutivos não dependem deste tipo de premissa. Fundamentalmente, a medição de performance deve ser capaz apenas de ordenar duas soluções comparativas e determinar aquela, que de alguma forma, é melhor que a outra (FOGEL, 2000). 
Os algoritmos genéticos são os modelos mais conhecidos, baseados na teoria da evolução natural e na genética, estes modelos de otimização possuem a capacidade de trabalhar problemas com uma grande gama de soluções. Nos algoritmos genéticos a população é um conjunto de possíveis soluções ao problema determinado, sendo cada indivíduo dessa população com uma estrutura semelhante aos cromossomos (Figura 4).

Figura 4 - Diagrama geral do ciclo de vida de um algoritmo genético

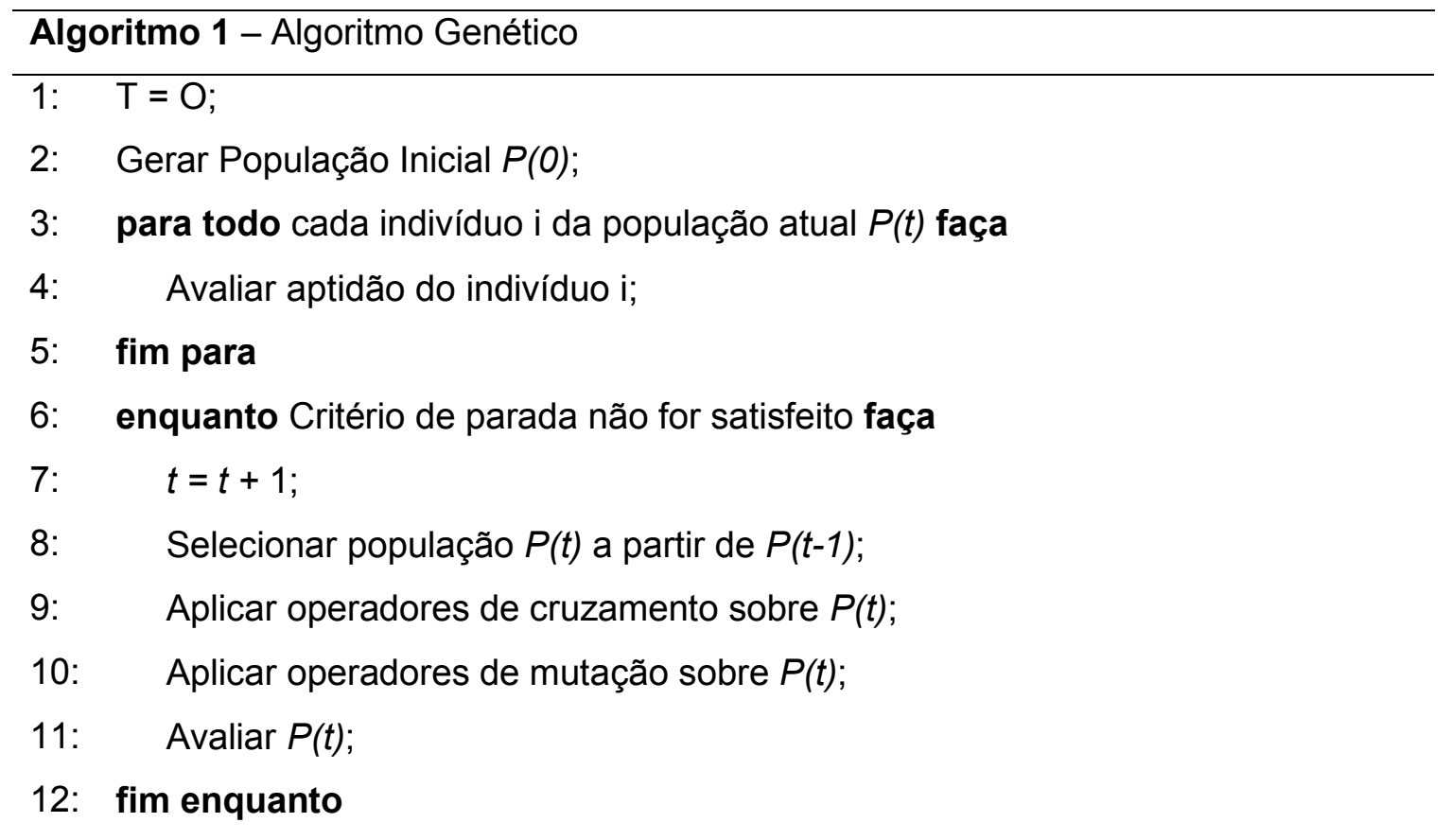

Fonte: Rezende (2005)

A possibilidade de sobrevivência de cada indivíduo é avaliada por uma função custo, função esta a ser otimizada, sendo o resultado desta função a aptidão de cada indivíduo como melhor resultado ao problema, funcionando de forma de seleção para reprodução. Finalmente a evolução é propiciada pela aplicação de operadores genéticos como seleção, cruzamento e mutação (MARTINEZ et al, 2009).

Os operadores de seleção busca averiguar o quão apto cada indivíduo está, para ser considerado a melhor solução ao problema encontrado, depois disso, os indivíduos são cruzados, ou seja, pela junção de partes de cada um dos indivíduos aptos é formada uma nova população de indivíduos e, eventualmente, alguns destes indivíduos sofrem alterações aleatórias de mutação, segundo uma determinada 
probabilidade de ocorrência.

Existem implicações no resultado final do modelo de acordo com os parâmetros e técnicas destes operadores, mas a função de seleção, que ordena os indivíduos mais aptos garante que as melhores alternativas encontradas ao problema sejam sempre mantidas.

\section{METODOLOGIA}

A metodologia deste trabalho está voltada ao desenvolvimento de algoritmos genéticos capazes de otimizar a definição do limite inferior no modelo de Miller e Orr. Nesse sentido, é necessário o desenvolvimento de simulações em diferentes cenários para obtenção de séries de fluxos líquidos de caixa que possibilite a validação do modelo desenvolvido.

No caso específico do problema abordado, autores já apresentados, destacaram a necessidade de não considerar o saldo de caixa como uma variável aleatória exclusivamente com distribuição normal. Dessa forma, investigar os resultados obtidos em simulações com diferentes características é de suma importância.

Assim, para obter dados que atendam a estes requisitos será utilizado o método de Monte Carlo ( $\mathrm{MC}$ ). O método de Monte Carlo consiste em um método numérico que permite resolver problemas matemáticos mediante a simulação de variáveis aleatórias (SÓBOL, 1976).

A geração de números pseudo-aleatórios pode ser realizada por diversas técnicas numéricas, de acordo com a função densidade probabilidade (f.d.p.) que se deseja. De acordo com a literatura apresentada os fluxos de caixa serão gerados segundo a Distribuição Normal, com média $\mu$ e variância $\sigma 2$ esta distribuição possui uma tendência central.

Dessa forma, foram definidos alguns aspectos da simulação, com a geração de nove fluxos de caixa com 500 valores cada, alterando a sua média e variância de acordo com três parâmetros (Tabela 1). Optou-se inicialmente pela média da Amostra 1 como sendo zero, pois neste caso existe a mesma probabilidade do fluxo de caixa possuir saldo negativo ou positivo. Nas demais amostras foram consideradas médias maiores (\$5.000 e \$20.000) para analisar casos com menores 
probabilidades de fluxo de caixa negativo.

Tabela 1 - Geração de números aleatórios

\begin{tabular}{lccc}
\hline Geração de Números Aleatórios & Média & Desvio-Padrão & Quantidade \\
\hline Amostra 1 (média = zero) & & & \\
Amostra 1.A & zero & 500 & 500 \\
Amostra 1.B & zero & 1500 & 500 \\
Amostra 1.C & zero & 2500 & 500 \\
Amostra 2 (média =5.000) & & & \\
Amostra 2.A & 5000 & 500 & 500 \\
Amostra 2.B & 5000 & 1500 & 500 \\
Amostra 2.C & 5000 & 2500 & 500 \\
Amostra 3 (média $=20.000)$ & & & \\
Amostra 3.A & 20000 & 500 & 500 \\
Amostra 3.B & 20000 & 1500 & 500 \\
Amostra 3.C & 20000 & 2500 & 500 \\
\hline
\end{tabular}

Fonte: Elaborado pelos autores

O objetivo foi validar o modelo de acordo com fluxos com médias e variâncias distintas, obtendo fluxos com valores negativos de saldo de caixa ou apenas com valores positivos.

A análise descritiva das séries de fluxos gerada apresenta o detalhamento das distribuições (Tabela 2). 
Tabela 2 - Análise descritiva das amostras

\begin{tabular}{llllllllll}
\hline $\begin{array}{l}\text { Análise } \\
\text { Descritiva } \\
\text { Amostras }\end{array}$ & $1 . A$ & $1 . B$ & $1 . C$ & $2 . A$ & $2 . B$ & $2 . C$ & $3 . A$ & $3 . B$ & $3 . C$ \\
\hline $\begin{array}{l}\text { Média } \\
\text { Desvio }\end{array}$ & 22,14 & 88,72 & 93,07 & 4953,35 & 5026,75 & 5194,54 & 19971,69 & 20065,03 & 19911,60 \\
padrão & 507,91 & 1544,65 & 2549,09 & 491,22 & 1453,51 & 2595,18 & 491,37 & 1476,85 & 2497,80 \\
Variância & & & & & & & & & \\
da & & & & & & & & & \\
amostra & $2,58 \mathrm{E}+05$ & $2,39 \mathrm{E}+06$ & $6,50 \mathrm{E}+06$ & $2,41 \mathrm{E}+05$ & $2,11 \mathrm{E}+06$ & $6,73 \mathrm{E}+06$ & $2,41 \mathrm{E}+05$ & $2,18 \mathrm{E}+06$ & $6,24 \mathrm{E}+06$ \\
Curtose & $-0,33$ & $-0,08$ & $-0,21$ & 0,17 & 0,67 & 0,35 & $-0,19$ & 0,05 & $-0,12$ \\
Assimetria & $-0,05$ & 0,02 & 0,00 & 0,10 & $-0,06$ & 0,02 & 0,16 & $-0,08$ & 0,07 \\
Intervalo & 2802,41 & 8792,27 & 15715,34 & 3002,35 & 9800,93 & 17357,52 & 2990,89 & 9339,38 & 13594,13 \\
Mínimo & $-1384,72$ & $-4366,67$ & $-7924,25$ & 3541,75 & $-140,90$ & $-3718,05$ & 18649,11 & 15693,25 & 13049,46 \\
Máximo & 1417,68 & 4425,60 & 7791,10 & 6544,10 & 9660,03 & 13639,47 & 21640,00 & 25032,63 & 26643,59 \\
Contagem & 500 & 500 & 500 & 500 & 500 & 500 & 500 & 500 & 500 \\
\hline
\end{tabular}

Fonte: Elaborado pelos autores

Em seguida, os fluxos foram aplicados ao AG programado para otimizar o parâmetro LM (limite mínimo) do modelo Miller-Orr com a função objetivo de minimizar o custo total.

Foram definidos dois modelos de algoritmos genéticos, o primeiro (AG1) com as definições normais e o segundo (AG2) com a supressão dos operadores de cruzamento, ou seja, seria utilizado apenas operadores de mutação

Os parâmetros definidos foram os seguintes:

- Saldo inicial de caixa: todas as séries de saldos de caixa partiram de um saldo inicial de $\$ 1.000,00$, somado a cada momento com o valor gerado na série de fluxos de caixa. A determinação de um saldo inicial fixo não intefere na relevância dos fluxos, pois é ajustado logo após o cálculo do primeiro fluxo de caixa;

- Custo de transação $(F)$ foi definido arbitrariamente em \$2,85 como uma tarifa incidente por operação, seja ela de investimento (saída de caixa para o investimento) quando o saldo atinge o limite superior, ou desinvestimento (saída do investimento para o caixa) quando o saldo atinge o limite mínimo estabelecido;

- Custo de oportunidade (K), assumido pelo custo financeiro de obter o 
valor da ruptura de caixa emprestado, de $1 \%$ sobre este valor;

- Limite mínimo (LM) que é o objetivo do AG deve ser obtido entre $\$ 2.000$ e $\$ 10.000$, sendo este intervalo definido arbitrariamente entre o limite de crédito que a empresa possui como sendo $\$ 2.000$ e $\$ 10.000$ em montante suficiente para cobrir seus riscos;

- Foram gerados 100 indivíduos LM para cada série simulada, com 500 iterações do AG para obtenção do Custo de cada fluxo de caixa e do Custo Total da série, com valores variando de $-\$ 2.000,00$ até $\$ 10.000,00$;

- Para os AG's foram definidos os parâmetros:

- Valores: binários, transformados a partir das séries de saldo de caixa (AG1 e AG2);

- Cruzamento: método da roleta entre 2 pais gerando 2 filhos (AG1);

- Mutação: taxa de mutação de $1 \%$, alterando um determinado bit do valor (AG1 e AG2).

Os parâmetros utilizados nesta metodologia foram atribuídos de maneira empírica, visando à observação da composição do resultado da otimização do valor de LM, pois não foram observadas referências que pudessem basear a forma de estruturação do referido problema de otimização. A seguir os resultados são apresentados e analisados.

\section{ANÁLISE DOS RESULTADOS}

Os resultados obtidos pela fórmula de otimização são apresentados na Tabela 3 
Tabela 3 - Resultados ótimos

\begin{tabular}{|c|c|c|c|c|}
\hline $\begin{array}{l}\text { AG1 - com Operadores } \\
\text { Geração de Números } \\
\text { Aleatórios }\end{array}$ & $\begin{array}{l}\text { zamento e Mutação } \\
\text { Limite Mínimo } \\
\text { Ideal }\end{array}$ & $\begin{array}{l}\text { Custo } \\
\text { Total }\end{array}$ & $\begin{array}{l}\text { Média } \\
\text { LM }\end{array}$ & $\begin{array}{c}\text { Desvio } \\
\text { Padrão LM }\end{array}$ \\
\hline \multicolumn{5}{|l|}{ Amostra 1 (média = 0) } \\
\hline Amostra 1.A & 2380,90 & 1508,68 & & \\
\hline Amostra 1.B & 5712,60 & 1150,25 & 5566,13 & 3114,58 \\
\hline Amostra 1.C & 8604,90 & 1226,72 & & \\
\hline \multicolumn{5}{|l|}{ Amostra 2 (média $=5.000$ ) } \\
\hline Amostra 2.A & 9801,90 & 868,85 & & \\
\hline Amostra 2.B & 9676,40 & 879,41 & 9775,07 & 88,36 \\
\hline Amostra 2.C & 9846,90 & 888,55 & & \\
\hline \multicolumn{5}{|c|}{ Amostra 3 (média $=20.000$ ) } \\
\hline Amostra 3.A & 8211,00 & 1068,75 & & \\
\hline Amostra 3.B & 9617,40 & 1065,90 & 9062,17 & 748,45 \\
\hline Amostra 3.C & 9358,10 & 1065,90 & & \\
\hline $\begin{array}{l}\text { AG2 - com Operadores } \\
\text { Geração de Números } \\
\text { Aleatórios }\end{array}$ & $\begin{array}{l}\text { ação } \\
\text { Limite Mínimo } \\
\text { Ideal }\end{array}$ & $\begin{array}{l}\text { Custo } \\
\text { Total }\end{array}$ & $\begin{array}{c}\text { Média } \\
\text { LM }\end{array}$ & $\begin{array}{c}\text { Desvio } \\
\text { Padrão LM }\end{array}$ \\
\hline \multicolumn{5}{|l|}{ Amostra 1 (média = 0) } \\
\hline Amostra 1.A & 2234,40 & 1507,21 & & \\
\hline Amostra 1.B & 5473,80 & 1129,47 & 5432,03 & 3176,96 \\
\hline Amostra 1.C & 8587,90 & 1226,55 & & \\
\hline \multicolumn{5}{|l|}{ Amostra 2 (média $=5.000$ ) } \\
\hline Amostra 2.A & 9860,00 & 866,58 & & \\
\hline Amostra 2.B & 9453,20 & 880,03 & 9737,17 & 246,67 \\
\hline Amostra 2.C & 9898,30 & 889,07 & & \\
\hline \multicolumn{5}{|c|}{ Amostra 3 (média $=20.000$ ) } \\
\hline Amostra 3.A & 9932,30 & 1065,90 & & \\
\hline Amostra 3.B & 9193,10 & 1065,90 & 9612,60 & 379,57 \\
\hline Amostra 3.C & 9712,40 & 1065,90 & & \\
\hline
\end{tabular}

Fonte: Elaborado pelos autores

Os resultados obtidos apontam para a definição de um limite mínimo ideal $\left(L^{*}\right)$ capaz de reduzir o Custo Total da manutenção de saldo em caixa. Neste ponto, analisar as amostras e seus resultados torna-se relevante.

Primeiro, nota-se que independente da utilização dos operadores de cruzamento, ambos algoritmos (AG1 e AG2) apresentaram resultados muito semelhantes, o que indica a prevalência dos operadores de mutação para garantir a ambrangência espacial do modelo, onde, normalmente, a utilização exclusiva dos operadores de cruzamento tende a uma otimização local (REZENDE, 2005). 
No caso das amostras 2 e 3 para os dois modelos, que apresentam médias maiores e menores chances de saldo negativo de caixa, mesmo com a mudança significativa do desvio padrão entre os subgrupos A, B e C, o valor ideal de $L^{*}$ * quase não obteve variações, destacando-se a amostra 2 com menor desvio padrão entre os valores de $\mathrm{LM}^{*}$ obtidos.

Dessa maneira, nota-se que os custos totais das amostras ficam próximos mesmo com uma maior variabilidade dos fluxos de caixa, no caso das amostras $2 \mathrm{e}$ 3 , avaliados pelo desvio padrão calculado dos três valores obtidos para $\mathrm{LM}^{*} \mathrm{em}$ cada grupo.

Já no caso da amostra 1, com média zero e consequentemente maiores chances de ruptura de caixa, os valores obtidos de $\mathrm{LM}^{*}$ apresentaram maiores diferenças, bem como um maior desvio padrão para os valores obtidos em $\mathrm{LM}^{*}$ de cada grupo.

Com isso, pode-se verificar que no caso de empresas cuja média do fluxo de caixa seja positiva, proporcionando um nível de caixa mais alto, o custo do caixa é reduzido, como é o caso da amostra 2. Mesmo assim, fluxos de caixa com médias altas tendem a elevar novamente o custo, conforme demonstra a amostra 3.

Dessa forma, no caso de empresas com fluxos de caixa baixos, como é o caso da amostra 1 (com a média do fluxo de caixa sendo zero), o risco de ruptura de caixa eleva os valores mínimos necessários $\left(L^{*}\right)$ conforme a variabilidade da amostra aumenta (1A, 1B e 1C), enquanto nas amostra com maior média (amostras 2 e 3) os valores de $\mathrm{LM}^{*}$ obtidos ficam bem próximos entre si, com uma baixa variabilidade demonstrada na coluna Desvio Padrão LM.

Os resultados obtidos como ótimos demonstram que os modelos foram capazes de chegar a um ponto de mínimo custo, devido a especificação dos AG's, com alta probabilidade de obter o mínimo global.

\section{CONSIDERAÇÕES FINAIS}

Os algoritmos genéticos têm se demonstrado uma ferramenta muito útil na aplicação deste tipo de problema de otimização. Ao auxiliarem na definição de parâmetros como o limite mínimo de caixa, pode-se encontrar com maior imparcialidade o valor ideal para o saldo de caixa. 
Os resultados apontam para uma área promissora, ainda assim, maiores estudos e simulações são necessárias, uma vez que os resultados não puderam ser comparados com outros modelos, assim, demanda-se um estudo mais aprofundado com a comparação com os modelos de definição do saldo de caixa mais recentes.

Apesar disso, não há métrica de comparação para os modelos aplicados, uma vez que o modelo original de Miller e Orr não soluciona a definição do ponto ideal de caixa, apenas o apresenta como sendo uma função da distribuição dos fluxos de caixa de acordo com o valor mínimo determinado (limite mínimo). Além disso, os algoritmos genéticos encontram o melhor valor, dentro de seu objetivo, com base experimental, assim, em alguns desses fluxos pode existir mais de um $\mathrm{LM}^{*}$, ou mesmo, novos experimentos podem encontrar soluções melhores.

O estudo contribui significativamente ao apontar a necessidade de operadores de mutação para o problema. Maiores simulações relativas a este operador podem indicar vantagens significativas na otimização do problema.

Os resultados apresentam vantagens, principalmente em um problema de otimização com grande variabilidade de respostas, impossível de ser resolvido por outras técnicas de otimização lineares, indicando uma metodologia consistente para o problema de otimização do saldo de caixa.

\section{REFERÊNCIAS}

ASSAF NETO, A. Finanças corporativas e valor. São Paulo: Atlas, 2005.

BACCARIN, S. Optimal impulse control for cash management with quadratic holdingpenalty costs. Decision in Economics and Finance, v. 25, p. 19-32, 2002.

BACCARIN, S. Optimal impulse control for a multidimensional cash management system with generalized cost functions. European Journal of Operational Research, v. 196, p. 198-206, 2009.

BAUMOL, W. The transaction demand for cash-an inventory theoretic approach. The Quarterly Journal of Economics, v. 66, p. 545-556, 1952.

BEDWORTH, D. D.; BAILEY, J. E. Integrated production, control systems: management, analysis and design. New York: John Wiley and Sons, 1987.

BERK, J.; DEMARZO, P. Finanças empresariais. São Paulo: Bookman, 2008. 
FOGEL, D.B. What is evolutionary computation? IEEE Spectrum, p. 26-32, fev., 2000.

GORMLEY, F.M.; MEADE, N. The utility of cash flow forecasts in the management of corporate cash balances. European Journal of Operational Research, v. 182, p. 923-935, 2007.

HINDERER, K.; WALDMANN, K-H. Cash management in randomly varying environment. European Journal of Operational Research, v. 130, p. 468-485, 2001.

LIU, B; XIN, C. An online model for managing cash: an alternative approach to the miller-orr model. Fourth International Conference on Natural Computation - IEEE Computer Society, 2008.

MATÍNEZ, M.; GARCÍA-NIETO, S.; SANCHIS, J.; BLASCO, X. Genetic algorithms optimization for normalized normal constraint method under pareto construction. Advances in Engineering Software, v. 40, p. 260-267, 2009.

MATIAS, A.B. (Org.). Finanças corporativas de curto prazo. São Paulo: Ed. Atlas, 2007.

MILBOURNE, R. Optimal money holding under uncertainty. International Economic Review, v. 24, n. 3, p. 685-698, 1983.

MILLER, M.; ORR, D. A model of the demand for money by firms. The Quarterly Journal of Economics, v. 81, p. 413-435, 1966.

PREMACHANDRA, I.M. A diffusion approximation model for managing cash in firms: an alternative approach to the miller-orr model. European Journal of Operational Research, v. 157, p. 218-226, 2004.

REZENDE, S.O. (Org.). Sistemas inteligentes: fundamentos e aplicações. São Paulo: Manole, 2005.

ROSS, S. A.; WESTERFIELD, R. W.; JAFFE, J. F. Administração financeira: corporate finance. São Paulo: Atlas, 2002.

SÁ, C. A. Fluxo de caixa: a visão da tesouraria e da controladoria. São Paulo: Atlas, 2008.

SLACK, N.; CHAMBERS, S.; HARLAND, C.; HARRISON, A.; JOHNSTON, R. Administração da produção. São Paulo: Atlas, 1997.

SÓBOL, L. M. Método de Monte Carlo. Ed. MIR, 1976.

SRINIVASAN, V.; KIM, Y. H. Deterministic cash flow management: state of art and research directions. OMEGA - International Journal of Management Sciences, v. 14, n. 2, p. 145-166, 1986. 
TAPIERO, C. S.; ZUCKERMAN, D. A Note on the optimal control of a cash balance problem. Journal of Banking and Finance, v. 4, p. 345-352, 1980.

VOLOSOV, K.; MITRA, G.; SPAGNOLO, F.; CARISMA, C. L. Treasury management model with foreign exchange exposure. Computational Optimization and Applications, v. 39, p. 179-207, 2005.

YAO, J-S.; CHEN, M-S.; LU, H-F. A fuzzy stochastic single-period model for cash management. European Journal of Operational Research, v. 170, p. 72-90, 2006.

ZHANG, D.; TSAI, J. J. P. Machine learning and software engineering. Software Quality Journal, v. 11, p. 87-119, 2003.

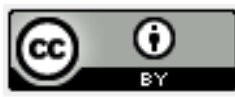

Artigo recebido em 14/04/2010 e aceito para publicação em 10/04/2011. 